\title{
Iatrogenic Dissection of Left Main Stem Coronary Artery Necessitating Emergency Coronary Artery Bypass Grafting: A Rare Life Threatening Complication of Diagnostic Coronary Angiography
}

\author{
Amer Harky*, Megan Garner, Alessia Rossi, Benjamin Adams, David Lawrence and Carmelo Di Salvo \\ Department of Cardiac Surgery, St. Bartholomew's Hospital, UK
}

Submission: April 03, 2017; Published: June 12, 2017

*Corresponding author: Amer Harky, Department of Cardiothoracic Surgery, Barts Heart Centre, St. Bartholomew's Hospital, 1st Floor KGV Building, West Smithfield, London, EC1A 7BE, Email: aaharky@gmail.com

\begin{abstract}
Coronary angiography is the investigation of choice to understand coronary anatomy. Although it is a generally safe procedure, it is not risk free. Coronary artery dissection is a rare condition. It can be either spontaneous or iatrogenic as a complication of angiography, angioplasty or cardiac surgery. We report the case of an iatrogenic dissection of the left main stem coronary artery with extension into the left anterior descending artery (LAD) secondary to an elective coronary angiogram. The complication was treated with emergency coronary artery bypass surgery with a left internal mammary artery to LAD anastamosis and long saphenous vein as graft conduits respectively. We aim to highlight the importance of prompt identification of such complication as well as the challenges in management with regards to coronary artery bypass surgery or bail out stenting.
\end{abstract}

Keywords: Left main stem dissection; Coronary artery bypass surgery; Coronary dissection; Coronary angiogram

\section{Case Report}

A 73-year-old female presented with a one month history of exertional dyspnea. She denied chest pain, syncope or palpitations. Her past medical history included well controlled systemic hypertension, non-insulin dependent diabetes mellitus and she was an ex-smoker. On examination she was in sinus rhythm and cardiovascular examination was unremarkable. She proceeded to coronary angiogram which was performed through a right radial artery approach. While performing the left side heart angiogram and injecting the contrast through the left coronary Ostia, an immediate dissection of the left main stem (LMS) coronary artery with extension into the left anterior descending (LAD) coronary artery was noted, (Figure 1). During this time the patient reported severe sharp chest pain radiating to her left shoulder with associated dyspnea.

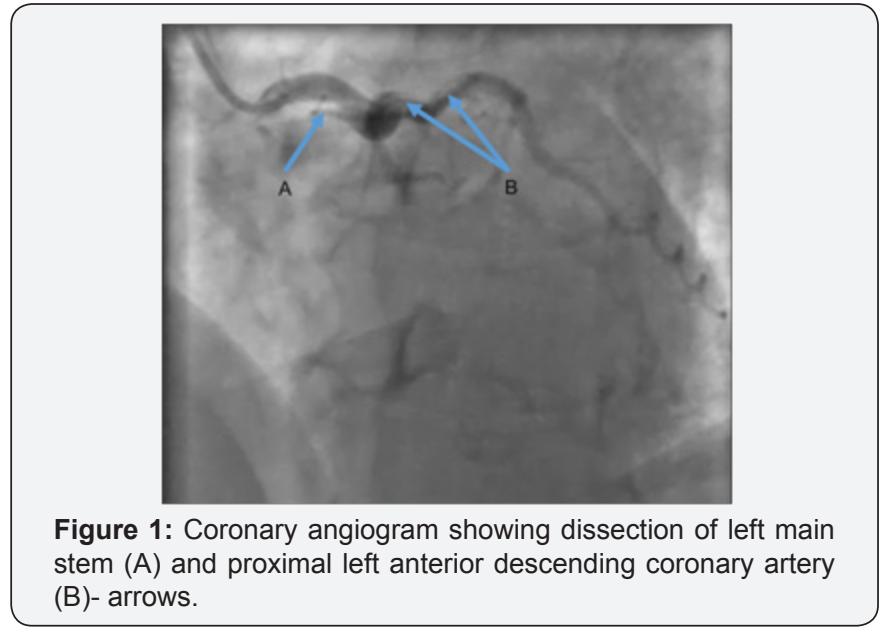

The procedure was abandoned and emergency advice was sought from the tertiary cardiac center. As the patient was haemodynamically stable with an extensive dissection 
involving the LMS and LAD a decision was made to transfer the patient for emergency coronary artery by pass grafting (CABG) surgery rather than consider bail-out stenting.

She underwent an emergency CABG using the left internal mammary artery to mid LAD and a saphenous vein graft to the inter mediate artery. The circumflex coronary artery was small and not-graft able; hence the intermediate coronary artery was grafted. Total cardiopulmonary bypass time was 52 minutes with aortic cross clamp time of 22 minutes. Her post-operative recovery was unremarkable and she was discharged home on day five post-surgery. Her post-operative transthoracic echocardiogram showed good left ventricular function.

\section{Discussion}

Iatrogenic dissection of the left main stem is a rare complication of coronary angiography with a reported incidence of $0.1 \%$ [1]. Such dissections can be classified according to the simplified Eshtehardi classification system where type I is a local dissection, type II involves the major branches of the LMS and type III which involves a dissection extending into the aortic root [2]. Increased risk of LMS dissection is thought to be associated with an acute angle take off of the left main stem, sub-intimal passage of the guide wire, atherosclerosis of the LMS and vigorous infusion of contrast [3].

Conservative management of these dissections is not advised due to the unpredictable nature of the dissection flap and the potential for significant deterioration [4]. Instead methods to seal the dissection flap are preferred, namely coronary artery bypass grafting (CABG) or percutaneous coronary intervention (PCI). Recommendations for the management of LMS dissection have been suggested by Esthardi in 2010 [4]. Here it is suggested that CABG is recommended in patients with extensive coronary artery disease which would require significant percutaneous intervention, an extended LMS dissection or widespread coronary artery disease in a haemodynamically stable patient. In our case the patient was haemodynamically stable with an extended LMS dissection which resulted in coronary artery bypass grafting being the treatment modality of choice. In unstable patients or those with a limited dissection such as type I, bailout PCI maybe considered. Where bailout stenting is used (with the backup of cardiothoracic surgical input in case of failure) survival rates remain encouraging, with Chen et al. [5] in 2008 reporting a survival rate of $94.4 \%$.

\section{Conclusion}

Careful interventional technique is the key to help prevent such a complication. Early recognition and intervention is important for this potentially life threatening complication. CABG is recommended in haemodynamically stable patients with widespread coronary artery disease or extensive LMS dissections.

\section{Acknowledgment}

$\mathrm{AH}$ and $\mathrm{MG}$ are co-authors of this case report, who drafted the case report, SF, LW and BA has reviewed and edited the manuscript. CDS is the responsible cardiac surgeon who operated on the patient.

\section{References}

1. Awadalla H, Sabet S, Sebaie A, Rosales O, Smalling R (2005) Catheter-induced left main dissection incidence, predisposition and therapeutic strategies experience from two sides of the hemisphere. J Invasive Cardiol 17(4): 233-236.

2. Rogers J, Lasala J (2004) Coronary artery dissection and perforation complicating percutaneous coronary intervention. J Invasive Cardiol 16(9): 493-499.

3. Celik M, Yuksel U, Yalcinkaya E, Gokoglan Y, Iyisoy A (2013) Conservative treatment of iatrogenic left main coronary artery dissection: report of two cases. Cardiovasc Diagn Ther 3(4): 244 246.

4. Eshtehardi P, Adorjan P, Togni M, Tevaearai H, Vogel R, et al. (2010) Cook S. Iatrogenic left main coronary artery dissection: Incidence, classification, management, and long-term follow-up. American Heart Journal 159(6): 1147-1153.

5. Cheng C, Wu C, Hsieh Y, Chen Y, Chen C, et al. (2008) Percutaneous coronary intervention for iatrogenic left main coronary artery dissection. Int J Cardiol 126(2): 177-182.

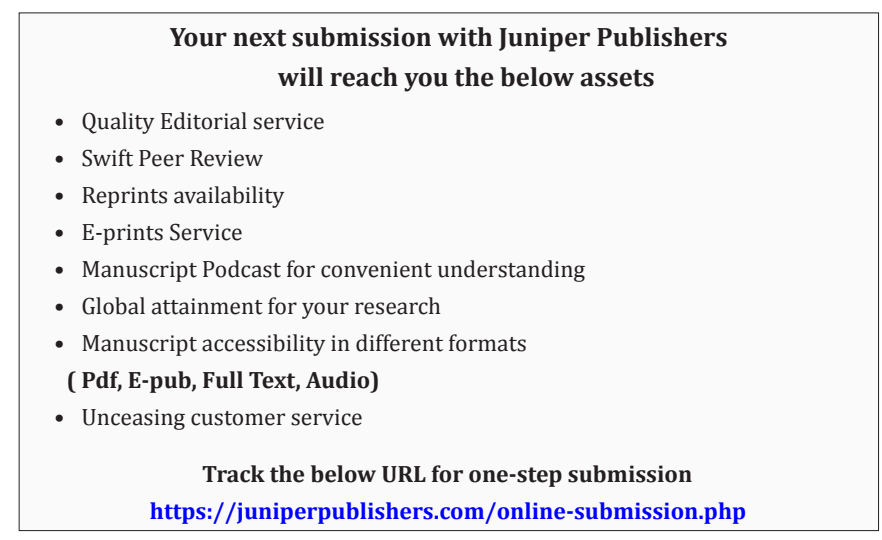

